\title{
Hopfield Neural Networks for Dynamic Channel Allocation in Mobile Communication Systems.
}

\author{
Enrico DEL RE, Romano FANTACCI, Luca RONGA, Giovanni GIAMBENE. \\ Dipartimento di Ingegneria Elettronica-Università di Firenze \\ Via S. Marta 3 - 50139 Firenze, Italy. \\ Tel. +39-55-4796270 - Fax +39-55-494569 - Telex 580681 UNFING-I
}

\begin{abstract}
The increasing demand of new services in the field of radio-mobile communications is in contrast with the capacity constraints imposed by the present communications medias. For this reason the use of techniques capable of ensuring that the spectrum assigned to mobile communications will be better exploited, is gaining an ever increasing importance. The search for an effective channel allocation technique along with the attempt to lower the computational costs of the algorithm which performs such a technique, was the aim to be achieved in our research.
\end{abstract}

\section{I - INTRODUCTION.}

The present challenge among communication researchers is to develop a worldwide Personal Communication Network (universal PCN) which, by enabling the reliable transfer of voice and data between two users traveling at any speed on land, sea, or in air, or located at any fixed position on earth, will be of great importance for the implementation of the new generation of PCSs.

In order to simplify the performance analysis of cellular systems, the territory is ideally divided into hexagonal cells. Each cell is managed by a Base Station (BS) with an omni-directional antenna located at its center.

The efficiency of a cellular system depends upon the possibility to use a given radio-channel in cells which are sufficiently far the one from the other, so that the co-channel interference is brought to acceptable levels (channel reuse). According to the multiple access technique employed by the cellular system, a channel is referred to as a fixed frequency bandwidth (FDMA), or a specific time-slot within a frame (TDMA) or a particular code (CDMA).

A channel cannot be used simultaneously in cells whose centers are closer than the reuse distance (D), which defines the interfering cells ring around each single cell of the cellular system.

Two typical alternatives to perform the channel assignment to the cells are the Fixed Channel Allocation Technique (FCA) and the Dynamic Channel Allocation Technique (DCA) [1]. While in the FCA a set of channels is permanently assigned to each cell, in the DCA the channels are assigned on a call-by-call basis in order to obtain a better performance and a narrower spectrum utilization, in particular under non-uniform traffic load conditions.

\section{II - THE DCA TECHNIQUE.}

In this paper a topological model is considered which is composed of a set of hexagonal cells forming a parallelogram whose sides have an equal number of cells.

Let $\mathrm{T}$ be the interference order; a cell $\mathbf{C}$ interferes with the cells belonging to $\mathrm{T}$ rings centered in $\mathbf{C}$.

The same channel cannot be used in two interfering cells: as this condition is inviolable, we refer to it as hard condition. Whenever the channel selected according to a suitable assignment algorithm does not satisfy this constraint, the corresponding service request (new call) is blocked. Other conditions, named soft conditions have also been provided. Soft conditions differ from hard conditions since they can be violated at the expense of a slight decrease in the allocation performance. The technique proposed in this paper is thought to be implemented in real cellular systems, so the number of operations to be performed is limited when a new call arrives. In particular, we do not consider any rearranging task in cells different from the one involved in the arrival as stated in [2].

Rearranging the assignment in the whole structure every time a call is received from the user, could obviously achieve a lower blocking probability; unfortunately, the amount of time needed to perform such a task makes this impossible to be realized in a practical cellular network.

The most important soft conditions proposed are the packing condition and the resonance condition.

With the packing condition, assignment solutions are preferred which tend to use the minimum number of channels to satisfy the global channel demand. The impact of this condition on the assignment is to prefer channels already used in other cells, without violating any hard condition. If more choices are possible, channels used in the nearest cells are taken into account. It will be explained later how to translate this and subsequent conditions into terms of a quadratic energy function to be minimized.

With the resonance condition, we tend to assign the same channels to cells that belong to the same reuse scheme which is obtained by jumping from one cell to another with steps of length exactly equal to the reuse distance, as shown in Fig. 1.

\footnotetext{
${ }^{1}$ This Work was carried out under the financial support of A.S.I. and M.U.R.S.T
} 

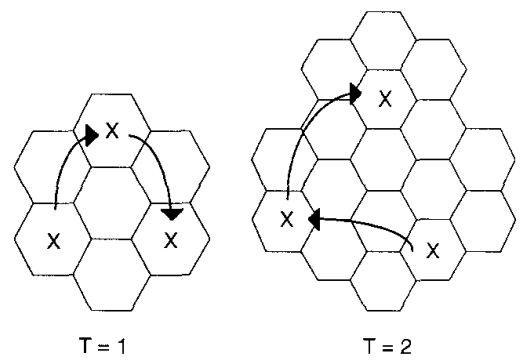

Fig. 1 - Reuse schemes.

This condition tends to give an optimum assignment in the presence of a uniform distribution of incoming calls among the cells. When a non-uniform traffic is present, this condition still seems to work well by arranging the assignment in an ordered way without interfering with the dynamic allocation concept.

Others soft conditions are formulated in order to:

- assign, where possible, the same channels assigned before, i.e. limiting the infracell rearranging;

- try to assign the exact number of channels requested in the cell involved in a new arrival (or in the termination of a call). A violation of this condition means the impossibility of serving an incoming call which is obviously blocked.

All these conditions lead to the definition of a quadratic energy function discussed below. In performing our analysis the following definitions are assumed:

CE............ the total number of cells in the system.

$\mathrm{CH}$........... the number of channels available to the system.

$\mathrm{A}_{\mathrm{i}, j} \ldots \ldots \ldots \ldots . . .$. an element of the allocation table whose value is $\mathbf{1}$ if channel $\mathrm{j}$ is allocated to cell $\mathrm{i}(\mathrm{i}=1, \mathrm{CE}$ and $\mathrm{j}=1, \mathrm{CH}), 0$ otherwise.

$i^{*}$.............. the cell involved in a new arrival or a termination of a call.

$\operatorname{Traf}\left(\mathrm{i}^{*}\right) \ldots . .$. the number of requested channels at cell $\mathrm{i}^{*}$.

$\mathrm{V}_{\mathrm{i}} * \mathrm{j} \ldots \ldots \ldots . .$. the assignment of the cell of interest; i.e. the variables of the assignment problem.

Interf( $\left(\mathrm{i}, i^{*}\right)$. the function giving a value of $\mathbf{1}$ if cells $\mathrm{i}$ and $i^{*}$ are interfering according to the previous definitions, $\mathbf{0}$ otherwise.

$\operatorname{Dist}\left(\mathrm{i}_{1} \mathrm{i}^{*}\right) \ldots$ this function gives the distance between cells $\mathrm{i}$ and $i *$ normalized to the inter-center distance between two adjacent cells.

$\operatorname{Res}\left(\mathrm{i}, \mathrm{i}^{*}\right) \ldots$. gives a value of $\mathbf{1}$ if cells $\mathrm{i}$ and $\mathrm{i}^{*}$ belong to the same reuse scheme defined before, 0 otherwise.

We define an energy function associated with the modeled cellular network as follows:

$$
\begin{aligned}
E & =\frac{A}{2} \cdot \sum_{j=1}^{C H} \sum_{\substack{i=1 \\
i \neq i^{*}}}^{C E} V_{i^{*}, j} \cdot A_{i, j} \cdot \operatorname{Interf}\left(i, i^{*}\right) \\
& +\frac{B}{2} \cdot\left(\sum_{j=1}^{C H} V_{i^{*}, j}-\operatorname{Traf}\left(i^{*}\right)\right)^{2} \\
& -\frac{C}{2} \cdot \sum_{j=1}^{C H} \sum_{\substack{i=1 \\
i \neq i^{*}}}^{C E} V_{i^{*}, j} \cdot A_{i, j} \cdot \frac{\left(1-\operatorname{Interf}\left(i, i^{*}\right)\right)}{\operatorname{Dist}\left(i, i^{*}\right)} \\
& -\frac{D}{2} \cdot \sum_{j=1}^{C H} V_{i^{*}, j} \cdot A_{i^{*}, j} \\
& +\frac{F}{2} \cdot \sum_{j=1}^{C H} \sum_{\substack{i=1 \\
i \neq i^{*}}}^{C E} V_{i^{*}, j} \cdot A_{i, j} \cdot\left(1-\operatorname{Res}\left(i, i^{*}\right)\right)
\end{aligned}
$$

The first term adds a positive constant to the energy function if there are some interfering cells using the same channels; the second term is positive if the requested number of channels has not been assigned to cell $i^{*}$; with the third term the packing condition is fulfilled as stated before; the fourth term lowers the value of the energy function if the actual assignment is equal to the previous one and the fifth term accomplishes the resonance condition.

Constants A, B, C, D, and $\mathrm{F}$ are determined in order to decide which are the conditions that can be violated and in what order, as is shown later.

Every time a new call arrives ( or ends) in a cell $i^{*}$, the algorithm searches for a pattern of 1 's and 0 's that, substituted to $\mathrm{V}_{\mathrm{i}^{*} \mathrm{j}}$, minimizes the energy function $E$. The best pattern found represents the solution to the DCA problem only if it satisfies the channel demand condition. On the contrary, if the pattern violates it, the arriving call is blocked.

With this formulation the problem complexity grows exponentially with the number of channels; hence we have proposed an approach based on Hopfield neural networks as described in the next section.

\section{III - HOPFIELD NEURAL NETWORKS.}

The combinatorial optimization problem, i.e. finding a solution that minimizes a cost function and whose variables assume only two possible values, falls in the more general class of NPcomplete (non-deterministic polynomial time complete) problems [3]. A neural network approach formulated by Hopfield and Tank [4] claimed to be a method that could be realized by hardware circuits with response times much shorter than those of other algorithmic methods.

Nevertheless, the original formulation suffered from a tendency to produce non-feasible solutions called "spurious states".

The variation proposed by Shigeo Abe [5] uses a particular formulation of Hopfield networks that eliminates all the spurious 
states from the solution space. This type of neural networks has been used in our simulations.

Giving an energy function :

$$
E=\frac{1}{2} x^{\prime} T x+b^{\prime} x
$$

where $T$ is a symmetric nxn matrix; $x$ is an n-elements vector representing our variable and $b$ is a constant $n$-elements vector of inputs. The problem is equal to finding $x$ that minimizes $E$ for every single component of $x$ with values 0 or 1 .

We extend the range of $x_{\mathbf{i}}$ - $\mathrm{i}$-th component of $x$ - to $[0,1]$.

Considering $x_{i}$ as the output of the $\mathrm{i}$-th neuron of the network, the internal state $u_{i}$ is introduced for which:

$$
x_{i}=f\left(u_{i}\right) \quad ; i=1 \ldots n
$$

where $f\left(u_{i}\right)$ is a monotone non linear function; for instance Hopfield suggests a hyperbolic tangent function $f\left(u_{i}\right)=1 / 2\left(1+\tanh \left(u_{i}\right)\right)$.

If we consider the quantity:

$$
\widetilde{E}=E-\sum_{i} \int_{0.5}^{x_{0}} f^{-1}(X) d X
$$

the temporal evolution of such an energy function is:

$$
\begin{aligned}
& \frac{d \widetilde{E}}{d t}=\sum_{i, j} T_{i, j} x_{j} \frac{d x_{i}}{d t}+\sum_{i} b_{i} \frac{d x_{i}}{d t}-\sum_{i} u_{i} \frac{d x_{i}}{d t} \\
& =\sum_{i} \frac{d x_{i}}{d t}\left(\sum_{j} T_{i, j} x_{j}+b_{i}-u_{i}\right)
\end{aligned}
$$

thus building a system for which:

$$
\frac{d u_{i}}{d t}=-\sum_{j} T_{i, j} x_{j}-b_{i}-u_{i}
$$

we have:

$$
\frac{d \tilde{E}}{d t}=-\sum_{i} \frac{d x_{i}}{d t} \frac{d u_{i}}{d t}=-\sum_{i}\left(\frac{d u_{i}}{d t}\right)^{2} \frac{d x_{i}}{d u_{i}} \leq 0
$$

for the monotonicity of $\mathrm{x}=\mathrm{f}(\mathrm{u})$. So $\widetilde{E}$ is a Lyapunov function for (4). Than we have:

$$
\frac{d \widetilde{E}}{d t} \leq 0
$$

confirming that the set of equations (4) leads E towards lower values; thus, for an arbitrary initial internal state $u_{0}$, a local minimum solution is achieved.

Under the hypothesis of using a piecewise-linear function as $f(u)$, system (4) becomes a linear system operating on a closed hypercube :

$$
\frac{d x}{d t}=-T x-b \quad 0 \leq x_{i} \leq 1 \quad i=1 \ldots n
$$

Its equilibrium points are all in the vertex of the hypercube and we can make all the feasible solutions be the only stable points [5].

It is not difficult to show that energy function $E$ in (1) can be expressed as a quadratic form of variables $V_{i * j}$ and if we call $\boldsymbol{x}$ the vector whose components are $V_{i *, j}$ with $j=1$.. $C H$ for a fixed $i^{*}$, expression (2) represents (1) with:

$$
\begin{aligned}
T_{j, j^{\prime}}= & B \\
b_{j}= & \frac{A}{2} \cdot \sum_{i} A_{i, j} \cdot \operatorname{Interf}\left(i, i^{*}\right) \\
& -B \cdot \operatorname{Traf}\left(i^{*}\right) \\
& -\frac{C}{2} \cdot \sum_{i} A_{i, j} \frac{\left(1-\delta_{i, i^{*}}\right)}{\operatorname{Dist}\left(i, i^{*}\right)}\left(1-\operatorname{Interf}\left(i, i^{*}\right)\right) \\
& -\frac{D}{2} \cdot A_{i^{*}, j} \\
& +\frac{F}{2} \cdot \sum_{i} A_{i, j} \cdot\left(1-\delta_{i, i^{*}}\right) \cdot\left(1-\operatorname{Res}\left(i, i^{*}\right)\right)
\end{aligned}
$$

where $\delta_{\mathrm{ij}}$ is the Kronecker delta.

In order to make system (6) stable for every possible $T$, we make the following substitutions without changing the values of $E$ at the vertexes of the hypercubic domain.

$$
\begin{gathered}
T_{j, j^{\prime}} \rightarrow 0 \quad \forall j \\
b_{j} \rightarrow b_{j}+\frac{T_{j, j^{\prime}}{ }^{(\text {old })}}{2}
\end{gathered}
$$

For the system under consideration it has been proven in [6] that no stable points exist within the variability domain. On the border of the hypercube there are vertexes and non vertexes points. The former are characterized by the fact that their components have only a value of 0 or 1 . The latter have at least one component in the interval $(0,1)$. Among vertexes, feasible solutions can be found which are compatible with the reuse and the channel demand constraints, and also unfeasible solutions.

The next step is to make all the feasible solutions be stable points of system (6).

Be $\mathbf{c}$ a vertex; $\mathbf{c}(\mathfrak{j})$ is an adjacent vertex obtained by substituting the $\mathrm{j}$-th component of $\mathrm{c}$ with its complement to 1 .

If $\mathbf{c}$ represents a feasible solution and $\mathrm{E}(\mathrm{c})$ is its energy value, in order to make $\mathbf{c}$ a strongly stable point we have to find values for constants A,B,C,D and F for which $E(\mathbf{c}(j))>E(c)$ for every $j$.

We decide whether a vertex represents a feasible solution on the basis of five rules: 


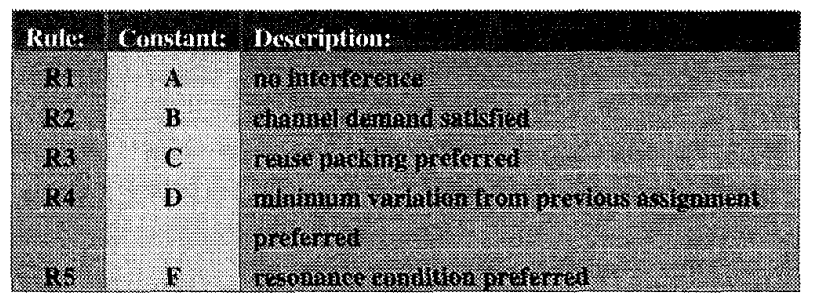

We start from a generic solution $\mathbf{c}$; then, we change one (or more) of its components from 0 to 1 or vice versa. Due to the priorities of our rules, we are interested in four types of transitions :

- transitions that violate rule $\mathrm{R} 1$, but lower energy for rules $\mathrm{R} 2, \mathrm{R} 3, \mathrm{R} 4$, and R5;

- transitions that increase energy for $\mathrm{R} 2$, but lower energy for $\mathrm{R} 3, \mathrm{R} 4$, and $\mathrm{R} 5$ leaving the term associated with $\mathrm{R} 1$ unchanged;

- transitions that increase energy for R5, but lower energy for $\mathrm{R} 3, \mathrm{R} 4$ leaving the terms associated with $\mathrm{R} 1$ and $\mathrm{R} 2$ unchanged;

- transitions that increase energy for R3, but lower energy for $\mathrm{R} 4$ leaving the terms associated with $\mathrm{R} 1, \mathrm{R} 2$, and $\mathrm{R} 5$ unchanged.

Since system (6) tends to lower energy during its evolution we have to find some relations among the constants of (1) so that each of the above named transitions coincides with an energy increment. These relations make all our feasible solutions be the only stable points in the system.

The relations are:

$$
\left\{\begin{array}{l}
C>D\left(\frac{1}{d_{1}}-\frac{1}{d_{2}}\right)^{-1} \\
E>\frac{C}{d_{\text {reuse }}}\left(\left[\frac{n_{\text {cells }}}{k_{\text {reuse }}}\right]-1\right)+D \\
B>E\left(\left[\frac{n_{\text {cells }}}{k_{\text {reuse }}}\right]\right) \\
B>\frac{C}{d_{\text {reuse }}}\left(\left[\frac{n_{\text {cells }}}{k_{\text {reuse }}}\right]-1\right)+D \\
A>B\left(2 n_{\text {channels }}+1\right)+\frac{C}{d_{\text {reuse }}}\left(\left\lceil\frac{n_{\text {cells }}}{k_{\text {reuse }}}\right]-1\right)+D
\end{array}\right.
$$

where $d_{\text {reuse }}$ and $k_{\text {reuse }}$ are the distance and reuse factors related to the reuse pattern of the cellular network.

In order to simulate the behavior of our neural network, we have built a software model of the Hopfield neural network using the Euler's method to solve the set of differential equations of (6). After one time step $\Delta \mathrm{t}$ we have:

$$
x_{i}^{\prime}=x_{i}-\Delta t\left\{T_{i} \cdot \bar{x}+b_{i}\right\}
$$

$$
\text { New } \quad x_{i}= \begin{cases}x_{i}^{\prime} & \text { for } 0<x_{i}^{\prime}<1 \\ 1 & \text { for } x_{i}^{\prime} \geq 1 \\ 0 & \text { for } x_{i}^{\prime} \leq 0\end{cases}
$$

where

$$
\begin{aligned}
& \left.\Delta t=\frac{R}{\operatorname{Max}_{i}\left\{\left(\frac{1}{2} T \cdot \overline{1}+b\right)\right\}}\right\} \\
& \overline{1}=[1,1,1,1,,, 1]^{\prime}
\end{aligned}
$$

For the initial value of $x$ we chose $x_{i}(0)=0.5+r_{i}$; where $r_{i}$ is a uniformly distributed pseudo-random value in the range $[-\alpha / 2$, $\alpha / 2]$.

In our simulation, we chose a value of $\mathrm{R}=0.3$ and $\alpha=10^{-9}$.

\section{IV - SIMULATIONS RESULTS.}

All the simulations have been carried out over a $7 \times 7$ portion of a hexagonal cell layout. We have considered two rings of interfering cells and 70 channels available to the whole system. In order to evaluate the performance of our technique, the blocking probability was used as a performance index.

The calls follow the Poisson arrival process with mean arrival rate equal to $\lambda$ (calls/min.). The call duration is an exponentially distributed statistical process with mean $\bar{x}$ (min.). The quantity $\bar{x} \cdot \lambda=\rho$ expresses the load offered to the cellular network.

We computed the probability of refusing an incoming call for the 9 central cells only and for a uniform traffic distribution among all the cells. Under these simulation hypotheses, we can compare our results with those obtained by Zhang and Yum [7] with the "Locally Optimized Dynamic Assignment" (LODA), "Borrowing with Channel Ordering" (BCO) and "Borrowing with Directional Channel Locking" (BDCL) allocation algorithms.

Curves for theoretical FCA (ERLANG-B) are added to the numerical results obtained from the simulations.

In Fig. 2, the behavior of our technique is shown with respect to others DCA techniques for a uniform traffic distribution. 


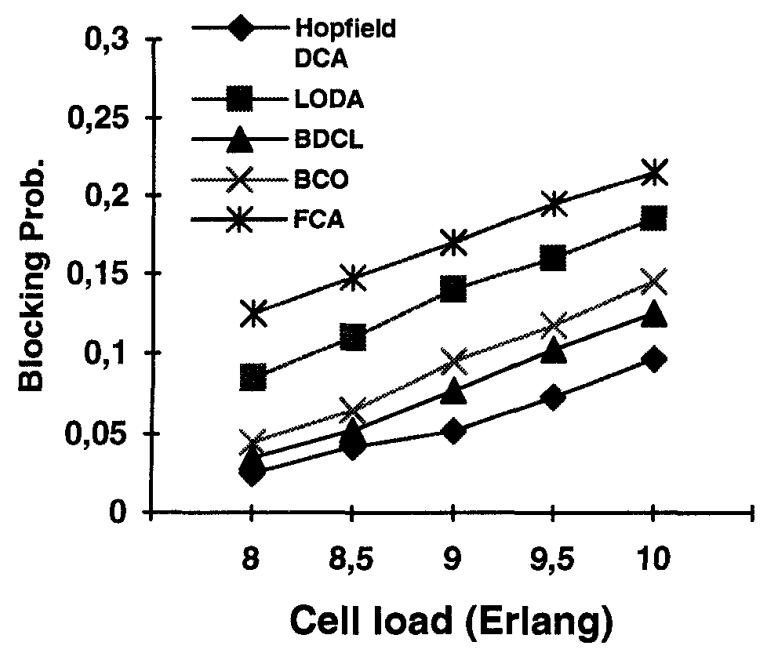

Fig. 2 - Performance with uniform traffic.

For a non-uniform traffic distribution the traffic pattern proposed by Zhang and Yum [7] shown in Fig.3 was considered.

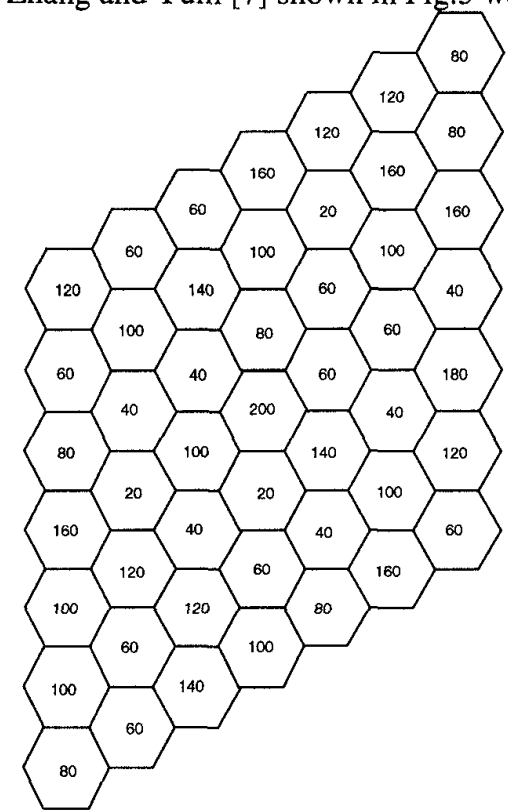

Fig. 3 - Non-uniform traffic distribution (calls per hour).

The traffic load for each cell increased by a percent factor ranging from 0 to 140 . The simulations results are summarized in Fig. 4.

\section{V - CONCLUSIONS.}

The structure of a cellular communication system suggests that a spread parallelized elaborative process for channel allocation is a natural choice. Another valuable feature of the abovenamed allocation technique is its insensitiveness to local faults; as a matter of fact, the loss of elaborative capacity of some neurons of the neural allocative network does not imply that the whole communication system will be blocked.
A neural approach like the one proposed in this paper seems to achieve a good level of performance with short processing times due to a massively parallel computational structure.

A further analysis of the Hopfield DCA allocator's behavior under different runtime conditions will be able to confirm the positive results derived by the simulations described in this paper.

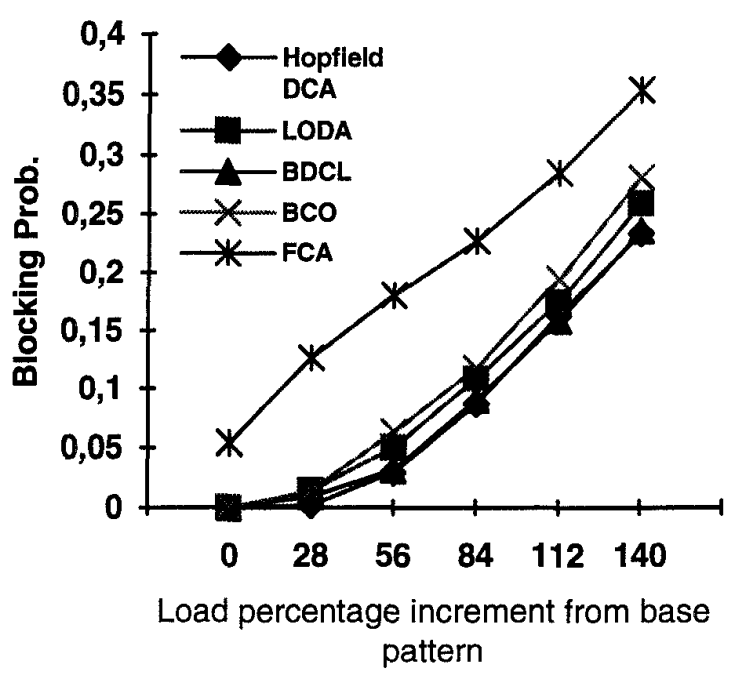

Fig. 4 - Performance with non uniform traffic load.

\section{REFERENCES.}

[1] W.C. Jakes, Jr., Microwave Mobile Communication, New York: Wiley, 1974.

[2] P.A. Raymond, "Performance Analysis of Cellular Networks", IEEE Trans. on Comm., Vol. 39, No. 12, Dec. 1991

[3] S. Kirkpatrick, C.D. Gelatt, M.P. De Vecchi, "Optimization by Simulated Annealing", Science, Vol. 220, No. 4598, May 1983

[4] J.J. Hopfield, D.W. Tank, "'Neural" Computation of Decisions in Optimization Problems", Biological Cybernetics, Vol. 52, pp. 141-152, 1982

[5] Shigeo Abe, "Global Convergence and Suppression of Spurious States of the Hopfield Neural Nets", IEEE Trans. Circuits and Systems, Vol. 40, No. 4, Apr. 1993.

[6] Jian-Hua Li, A. N. Michel, W. Porod, "Analysis and Syntesis of a Class of Neural Networks: Linear Systems Operating on a Closed Hypercube", IEEE Trans. Circuits and Systems, Vol. 36, No.11, Nov. 1989

[7] Ming Zhang, Tak-Shing P. Yum, "Comparison of Channel-Assignment Strategies in Cellular Mobile Telephone Systems", Trans. on Vehicular Tech., Vol.38, No. 4, Nov. 1989 